Bull. Soc. math. France

130 (1), 2002, p. 49-69

\title{
COHOMOLOGIE DE HOCHSCHILD DES GRAPHES DE KONTSEVICH
}

\author{
Par Didier Arnal \& Mohsen Masmoudi
}

\begin{abstract}
RÉsumÉ. - Nous calculons la cohomologie de Hochschild directement sur les graphes de Kontsevich. Celle-ci est localisée sur les graphes totalement antisymétriques ayant autant de pieds que de pattes. La considération de cette cohomologie permet de réinterpréter l'équation de formalité pour l'espace $\mathbb{R}^{d}$.

ABSTRACT (Hochschild cohomology of Kontsevich graphs). - We determine the Hochschild cohomology for the Kontsevich's graphs. As usual, that cohomology is localized on totally antisymmetric graphs with as many feet as legs. Using this cohomology, we reinterpret the formality equation for the space $\mathbb{R}^{d}$.
\end{abstract}

\section{Introduction}

L'opérateur de cohomologie de Hochschild (noté $d_{H}$ ) des opérateurs multidifférentiels apparaît naturellement dans l'équation de formalité de Kontsevich (voir [4] et sa formulation dans [2]) :

Texte reçu le 20 juillet 2000, accepté le 19 décembre 2000

DidiER ARnAL, Laboratoire de mathématiques MMAS de l'université de Metz,

UpresA CNRS 7035, Île du Saulcy 57045 Metz Cedex (France)

E-mail : arnal@poncelet.sciences.univ-metz.fr

Mohsen MAsmoudi, Laboratoire de mathématiques MMAS de l'université de Metz, UpresA CNRS 7035, Île du Saulcy 57045 Metz Cedex (France)

E-mail : masmoudi@poncelet.sciences.univ-metz.fr

Classification mathématique par sujets (2000). — 46L65, 16E40, 05C90.

Mots clefs. - Formalité, cohomolgie de Hochschild, graphes. 


$$
\begin{aligned}
0= & -d_{H}\left(\mathcal{F}_{k_{1}, \ldots, k_{n}}\left(\alpha_{1} \otimes \cdots \otimes \alpha_{n}\right)\right) \\
+ & \frac{1}{2} \sum_{\substack{I \sqcup J=\{1, \ldots, n\} \\
I, J \neq \varnothing}} \varepsilon_{\alpha}(I, J)(-1)^{\left(\left|k_{I}\right|-1\right)\left|k_{J}\right|}\left[\mathcal{F}_{k_{I}}\left(\alpha_{I}\right), \mathcal{F}_{k_{J}}\left(\alpha_{J}\right)\right]_{G} \\
- & \frac{1}{2} \sum_{i \neq j} \varepsilon_{\alpha}(i, j, 1, \ldots, \widehat{i, j}, \ldots, n)(-1)^{k_{i}-1} \mathcal{F}_{\left(\left(k_{i}+k_{j}-1\right), k_{1}, \ldots, \widehat{k_{i}, k_{j}}, \ldots, k_{n}\right)} \\
& \left(\left[\alpha_{i}, \alpha_{j}\right]_{S} \otimes \alpha_{1} \otimes \cdots \otimes \widehat{\alpha_{i}, \alpha_{j}} \otimes \cdots \otimes \alpha_{n}\right)
\end{aligned}
$$

si $[,]_{G}$ est le crochet de Gerstenhaber et $[,]_{S}$ celui de Schouten. On peut écrire cette équation sous la forme :

$$
d_{H}\left(\mathcal{F}_{(n)}\right)= \pm \frac{1}{2} \sum_{i=1}^{n-1}\left[\mathcal{F}_{(i)}, \mathcal{F}_{(n-i)}\right]_{G} \mp \frac{1}{2} \sum_{i, j} \mathcal{F}_{(n-1)}\left(\left[\mathfrak{\bullet}_{i}, \bullet_{j}\right]_{S} \otimes \cdots \otimes \bullet\right) .
$$

Si on impose que $\mathcal{F}_{(1)}$ est juste l'opération qui identifie un $m$ tenseur $\alpha$ à l'opérateur $m$-différentiel d'ordre $1, \ldots, 1$ qu'il définit canoniquement :

$$
\mathcal{F}_{(1)}(\alpha)\left(f_{1}, \ldots, f_{m}\right)=\left\langle\alpha, d f_{1} \wedge \cdots \wedge d f_{m}\right\rangle,
$$

on peut chercher à construire toutes les formalités sur $\mathbb{R}^{\bullet}$ par induction sur $n$.

Si $\mathcal{F}_{(1)}, \ldots, \mathcal{F}_{(n-1)}$ ont été trouvés, le second membre de l'équation est alors un cocycle de Hochschild, la résolution de l'équation pour $n$ est assurée si ce cocycle est exact.

Comme la formalité de Kontsevich est une somme d'opérateurs construits à partir de graphes admissibles, on peut se ramener à un calcul cohomologique sur les graphes eux-mêmes. C'est ce que nous avions fait dans [1] où nous avons introduit la notion de cohomologie de Hochschild des graphes de Kontsevich, en nous restreignant au cas des bons graphes, liés aux structures de Poisson linéaires.

Le but du présent article est le calcul complet de tous les groupes de cohomologie de Hochschild des graphes de Kontsevich ou plutôt des combinaisons linéaires de graphes, en toute dimension et pour tous les graphes.

Ceci sera mené à bien en utilisant une suite spectrale classique dans ce genre de problèmes. Nous avons suivi l'approche de [3] pour la description de cette suite spectrale.

\section{Graphes orientés et opérateurs multidifférentiels}

Rappelons d'abord comment M. Kontsevich dans [4] associe à chaque graphe orienté $(\Gamma, \mathcal{O})$ un opérateur multidifférentiel $B_{(\Gamma, \mathcal{O})}$ défini sur $\mathbb{R}^{d}$ pour tout $d$ (on dira qu'il est défini sur $\mathbb{R}^{\bullet}$ ).

Pour définir un graphe $\Gamma$, on se donne d'abord ses sommets, qui sont de deux catégories : les sommets aériens sont des points $p_{j}(1 \leq j \leq n)$ du demi plan

TOME $130-2002-\mathrm{N}^{\mathrm{O}} 1$ 
de Poincaré

$$
\mathcal{H}=\{z \in \mathbb{C}, \mathfrak{I m}(z)>0\}
$$

les sommets terrestres sont eux des points $q_{\ell}$ de la droite réelle. On suppose toujours $q_{1}<q_{2}<\cdots<q_{m}$. On note $P$ l'ensemble des sommets aériens et $Q$ l'ensemble des sommets terrestres. Les arêtes sont des flèches $\overrightarrow{a b}$ partant d'un sommet aérien $a$ et arrivant sur un sommet aérien ou terrestre $b$. Il n'y a pas d'arête multiple. L'ensemble de tous les graphes ayant pour sommets les points $p_{j}$ et $q_{\ell}$ est noté

$$
G_{P, Q}=G_{\left\{p_{1}, \ldots, p_{n}\right\},\left\{q_{1}, \ldots, q_{m}\right\}}
$$

Si $\Gamma$ est un graphe, on note $\operatorname{Deb}\left(p_{j}\right)$ l'ensemble des flèches partant du sommet $p_{j}$ et $k_{j}$ le cardinal de $\operatorname{Deb}\left(p_{j}\right)$. On note aussi, pour chaque sommet $b$ de $\Gamma$, Fin $(b)$ l'ensemble des flèches aboutissant sur $b$ et $g_{b}$ son cardinal.

Le graphe $\Gamma$ de $G_{P, Q}$ étant donné, on l'oriente en choisissant un ordre total sur l'ensemble $\mathcal{A}(\Gamma)$ de ses arêtes. On note $\mathcal{O}$ cette orientation, $(\Gamma, \mathcal{O})$ le graphe orienté et $\overrightarrow{G_{P, Q}}$ l'ensemble de tous les graphes orientés.

Chaque graphe orienté $(\Gamma, \mathcal{O})$ permet de définir une application $B_{(\Gamma, \mathcal{O})}$ qui, à chaque famille $\alpha_{1}, \ldots, \alpha_{n}$ de tenseurs contravariants, totalement antisymétriques et d'ordres respectifs $k_{1}, \ldots, k_{n}$ sur $\mathbb{R}^{\bullet}$, associe un opérateur $m$ différentiel $B_{(\Gamma, \mathcal{O})}\left(\alpha_{1} \otimes \cdots \otimes \alpha_{n}\right)$. Les arêtes de $\Gamma$ sont d'abord numérotées suivant leur ordre de 1 à $\left|k_{\{1, \ldots, n\}}\right|=k_{1}+\cdots+k_{n}$ et on pose $K=\left|k_{\{1, \ldots, n\}}\right|$, ensuite on considère tous les multi-indices $\left(i_{1}, \ldots, i_{K}\right)$ variant entre 1 et la dimension $d$ de l'espace et on note $\alpha_{j}\left(\operatorname{Deb}\left(p_{j}\right)\right)$ la composante $\alpha_{j}^{i_{r_{1}} \cdots i_{r_{k_{j}}}}$ du tenseur $\alpha_{j}$ si l'ensemble $\operatorname{Deb}\left(p_{j}\right)$ est formé des arêtes numérotées $r_{1}<\cdots<r_{k_{j}}$ et $\partial_{\mathrm{Fin}(b)}$ l'opérateur

$$
\partial_{\operatorname{Fin}(b)}=\frac{\partial^{g_{b}}}{\partial x^{i_{s_{1}}} \cdots \partial x^{i_{s_{b}}}}
$$

si $\operatorname{Fin}(b)$ contient les arêtes numérotées $s_{1}, \ldots, s_{g_{b}}$. Alors $B_{(\Gamma, \mathcal{O})}\left(\alpha_{1} \otimes \cdots \otimes \alpha_{n}\right)$ est défini par

$$
\begin{array}{r}
B_{(\Gamma, \mathcal{O})}\left(\alpha_{1} \otimes \cdots \otimes \alpha_{n}\right)\left(f_{1}, \ldots, f_{m}\right)=\sum_{1 \leq i_{1}, \ldots, i_{K} \leq d} \prod_{j=1}^{n} \partial_{\operatorname{Fin}\left(p_{j}\right)} \alpha_{j}\left(\operatorname{Deb}\left(p_{j}\right)\right) \\
\times \prod_{l=1}^{m} \partial_{\operatorname{Fin}\left(q_{\ell}\right)} f_{\ell} .
\end{array}
$$

On étend enfin l'application $B$ linéairement à l'espace vectoriel des combinaisons linéaires

de graphes orientés $(\Gamma, \mathcal{O})$ en posant

$$
\gamma=\sum a_{(\Gamma, \mathcal{O})}(\Gamma, \mathcal{O})
$$

$$
B_{\gamma}=\sum a_{(\Gamma, \mathcal{O})} B_{(\Gamma, \mathcal{O})} .
$$

BULletin DE LA SOCIÉtÉ MATHÉMATIQUE DE FRANCE 


\section{Graphes non orientés}

Sur la formule ci-dessus, on voit que si l'on garde le graphe $\Gamma$ mais qu'on change son orientation en remplaçant $\mathcal{O}$ par $\mathcal{O}^{\prime}$, on obtient le même opérateur multidifférentiel à un signe près $\varepsilon_{\Gamma}\left(\mathcal{O}, \mathcal{O}^{\prime}\right)$ qui ne dépend que de $\Gamma$ et de ses orientations. Par exemple les deux orientations $\mathcal{O}$ et $\mathcal{O}^{\prime}$ ci-dessous du même graphe $\Gamma$
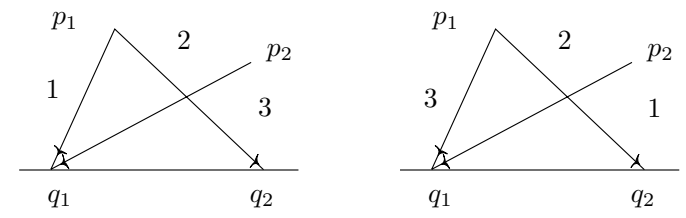

FiguRe 1

définissent les opérateurs

$$
\begin{aligned}
& B_{(\Gamma, \mathcal{O})}\left(\alpha_{1} \otimes \alpha_{2}\right)\left(f_{1}, f_{2}\right)=\sum_{1 \leq i_{1}, i_{2}, i_{3} \leq d} \alpha_{1}^{i_{1} i_{2}} \alpha_{2}^{i_{3}} \partial_{i_{1} i_{3}} f_{1} \partial_{i_{2}} f_{2}, \\
& B_{\left(\Gamma, \mathcal{O}^{\prime}\right)}\left(\alpha_{1} \otimes \alpha_{2}\right)\left(f_{1}, f_{2}\right)=\sum_{1 \leq i_{1}, i_{2}, i_{3} \leq d} \alpha_{1}^{i_{2} i_{3}} \alpha_{2}^{i_{1}} \partial_{i_{1} i_{3}} f_{1} \partial_{i_{2}} f_{2} .
\end{aligned}
$$

On a donc ici $\varepsilon_{\Gamma}\left(\mathcal{O}, \mathcal{O}^{\prime}\right)=-1$.

Comme on veut travailler seulement sur des graphes sans orientation, on ne garde que les combinaisons linéaires de graphes $\gamma$ telles que

$$
a_{\left(\Gamma, \mathcal{O}^{\prime}\right)}=\varepsilon_{\Gamma}\left(\mathcal{O}^{\prime}, \mathcal{O}\right) a_{(\Gamma, \mathcal{O})}
$$

pour tout couple d'orientations $\mathcal{O}$ et $\mathcal{O}^{\prime}$. Prenons alors une orientation « canonique » $\mathcal{O}_{0}$ d'un graphe $\Gamma$, par exemple l'ordre lexicographique des flèches $\overrightarrow{a b}$ de $\mathcal{A}(\Gamma)$, et notons $b_{\Gamma}$ le nombre $a_{\left(\Gamma, \mathcal{O}_{0}\right)} \# \mathcal{A}(\Gamma)$ ! et $B_{\Gamma}$ l'application $B_{\left(\Gamma, \mathcal{O}_{0}\right)}$. Si $O(\Gamma)$ désigne tous les ordres possibles sur $\mathcal{A}(\Gamma)$, on aura donc :

$$
\begin{aligned}
\gamma & =\sum_{\Gamma \in G_{P, Q}} \sum_{\mathcal{O} \in O(\Gamma)} a_{(\Gamma, \mathcal{O})}(\Gamma, \mathcal{O}) \\
& =\sum_{\Gamma \in G_{P, Q}} a_{\left(\Gamma, \mathcal{O}_{0}\right)} \sum_{\mathcal{O} \in O(\Gamma)} \varepsilon_{\Gamma}\left(\mathcal{O}, \mathcal{O}_{0}\right)(\Gamma, \mathcal{O})=\sum_{\Gamma \in G_{P, Q}} b_{\Gamma} e_{\Gamma}
\end{aligned}
$$

si

$$
e_{\Gamma}=\frac{1}{\# \mathcal{A}(\Gamma) !} \sum_{\mathcal{O} \in O(\Gamma)} \varepsilon_{\Gamma}\left(\mathcal{O}, \mathcal{O}_{0}\right)(\Gamma, \mathcal{O}) \quad\left(\text { alors } \quad B_{e_{\Gamma}}=B_{\left(\Gamma, \mathcal{O}_{0}\right)}\right)
$$

TOME $130-2002-\mathrm{N}^{\mathrm{O}} 1$ 
On notera simplement :

$$
\gamma=\sum_{\Gamma \in G_{P, Q}} b_{\Gamma} \Gamma, \quad B_{\gamma}=B_{\sum b_{\Gamma} \Gamma}=\sum_{\Gamma \in G_{P, Q}} b_{\Gamma} B_{\Gamma} \quad\left(=\sum_{\Gamma \in G_{P, Q}} b_{\Gamma} B_{\left(\Gamma, \mathcal{O}_{0}\right)}\right) .
$$

On identifiera donc l'espace ainsi obtenu à l'espace $V_{P, Q}$ engendré par les graphes non orientés et l'application $B$ est maintenant définie sur cet espace.

DÉfinition (opérateur de Kontsevich). - Soit $T\left(\mathbb{R}^{d}\right)$ l'espace des tenseurs contravariants totalement antisymétriques et $D\left(\mathbb{R}^{d}\right)$ celui des opérateurs différentiels sur $\mathbb{R}^{d}$. On appellera opérateur de Kontsevich l'application

$$
B: V_{P, Q} \longrightarrow \operatorname{End}\left(\bigotimes^{n} T\left(\mathbb{R}^{\bullet}\right), \bigotimes^{m} D\left(\mathbb{R}^{\bullet}\right)\right), \quad \gamma \longmapsto B_{\gamma} .
$$

REMARQUE. - La formalité de Kontsevich doit satisfaire une symétrie supplémentaire qui permette de remplacer le produit tensoriel $\alpha_{1} \otimes \cdots \otimes \alpha_{n}$ par un produit gradué symétrique $\alpha_{1} \cdots \cdots \alpha_{n}$ (voir par exemple [2]). Il faut donc considérer aussi l'action du groupe $\mathfrak{S}_{n}$ des permutations de $\{1, \ldots, n\}$ sur les graphes $\Gamma$, en permutant l'ordre des sommets aériens de $\Gamma$. Si on a muni $\Gamma$ de l'ordre canonique $\mathcal{O}_{0}$ et si $\sigma$ appartient à $\mathfrak{S}_{n}$, il faut alors distinguer l'opération qui consiste à permuter les flèches issues des sommets $p_{j}$ «par paquets », en conservant l'ordre des $\operatorname{Deb}\left(p_{j}\right)$ qu'on notera $\left(\mathcal{O}_{0}\right)^{\sigma}$ de l'ordre canonique du graphe $\Gamma^{\sigma}$ qu'on notera $\left(\mathcal{O}^{\sigma}\right)_{0}$. On notera $\varepsilon_{\Gamma}(\sigma)$ le signe $\varepsilon\left(\left(\mathcal{O}^{\sigma}\right)_{0}\left(\mathcal{O}_{0}\right)^{\sigma}\right) \cdot \varepsilon_{\alpha}(\sigma)$, où $\varepsilon_{\alpha}(\sigma)$ est défini dans [2] comme la signature de l'effet sur les $k_{j}$ impairs de la permutation $\sigma$.

Dans ce cas, on ne gardera finalement que les combinaisons linéaires de graphes non orientés $\gamma=\sum b_{\Gamma} \Gamma$ telles que $b_{\Gamma^{\sigma}}=\varepsilon_{\Gamma}(\sigma) b_{\Gamma}$ pour tout $\Gamma$ et tout $\sigma$. L'espace de ces combinaisons de graphes sera noté $V_{n, m}$, il est engendré par l'ensemble $G_{n, m}$ des classes $[\Gamma]$ de graphes $\Gamma$ à l'ordre des sommets aériens près.

Dans la suite, on ne tiendra pas compte de cette action de $\mathfrak{S}_{n}$.

\section{Pieds et pattes des graphes}

Soit $\Gamma$ un graphe non orienté, on appellera pieds de $\Gamma$ les sommets terrestres $q_{1}, \ldots, q_{m}$, pattes de $\Gamma$ les arêtes aboutissant à un pied, c'est-à-dire les éléments de $\operatorname{Fin}\left(q_{1}\right) \cup \cdots \cup \operatorname{Fin}\left(q_{m}\right)$. On notera $\Delta(\Gamma)$ le graphe purement aérien obtenu en supprimant la droite réelle, les pieds et les pattes de $\Gamma$ et $\partial \Delta(\Gamma)$ l'ensemble des origines des pattes de $\Gamma$. On représente les éléments de $\partial \Delta(\Gamma)$ par des couples $\left(p_{j}, x\right)\left(1 \leq x \leq r_{j}\right)$ en numérotant pour chaque sommet aérien $p_{j}$ les $r_{j}$ pattes qui en sont issues. Connaissant $\Delta=\Delta(\Gamma)$ et son bord $\partial \Delta=\partial \Delta(\Gamma)$ qui est une partie finie de $P \times \mathbb{N}$, on peut retrouver $\Gamma$ en se donnant une famille de parties $U_{1}^{\prime}, \ldots, U_{m}^{\prime}$ de $\partial \Delta$ telle que les $U_{\ell}^{\prime}$ non vides forment une partition de $\partial \Delta(\Gamma)$. Un tel graphe n'est admissible que si aucun $U_{\ell}^{\prime}$ ne contient deux 
éléments $\left(p_{j}, x\right)$ et $\left(p_{j}, y\right)$ avec le même $p_{j}$ et on met implicitement sur ses arêtes l'ordre canonique $\mathcal{O}_{0}$. On peut donc reconstruire les $U_{\ell}^{\prime} \subset P \times \mathbb{N}$ en connaissant leurs projections $U_{\ell}$ dans $P$ (si $p_{j} \in U_{\ell}$, il y aura dans $U_{\ell}^{\prime}$ l'élément $\left(p_{j}, k_{j \ell}\right)$ où $k_{j \ell}$ est le nombre des $\ell^{\prime} \leq \ell$ tels que $p_{j}$ appartient à $\left.U_{\ell^{\prime}}\right)$. On notera donc :

$$
\Gamma=\Gamma\left(\Delta, \partial \Delta,\left(U_{1}, \ldots, U_{m}\right)\right)=\Gamma(\Delta, \partial \Delta, \mathcal{U})=\Gamma_{\mathcal{U}}^{\Delta, \partial \Delta} .
$$

On sous-entendra même l'exposant, si $\Delta$ et $\partial \Delta$ sont fixés. D'ailleurs, il est clair que si l'on se donne $\Delta$ et $\mathcal{U}=\left(U_{1}, \ldots, U_{m}\right)$, le bord $\partial \Delta$ est complètement déterminé :

$$
\partial \Delta=\left\{\left(p_{j}, x\right), p_{j} \in U_{1} \cup \cdots \cup U_{m}, 1 \leq x \leq \#\left\{\ell \text { tels que } p_{j} \in U_{\ell}\right\}\right\} .
$$

On notera $\partial_{\mathcal{U}} \Delta$ ce bord.

Avec ces notations, l'opérateur $\Gamma \mapsto B_{\Gamma}=B_{\left(\Gamma, \mathcal{O}_{0}\right)}$ s'écrit :

$$
B_{\Gamma}\left(\alpha_{1} \otimes \cdots \otimes \alpha_{n}\right)\left(f_{1}, \ldots, f_{m}\right)=C_{\Delta, \partial \Delta}\left(\alpha_{1} \otimes \cdots \otimes \alpha_{n}\right) \partial_{U_{1}} f_{1} \cdots \partial_{U_{m}} f_{m} .
$$

En effet, les parties $U_{\ell}$ n'interviennent que dans les opérateurs de dérivation sur les $f_{\ell}$. Notons $D(P)$ l'ensemble des couples $(\Delta, \partial \Delta)$ admissibles construits sur $P$. Sur $V_{P, Q}, B$ s'écrira donc :

$$
\begin{aligned}
& \gamma=\sum_{\Gamma \in G_{P, Q}} b_{\Gamma} \Gamma=\sum_{(\Delta, \partial \Delta) \in D(P)} \sum_{\mathcal{U}} b_{(\Delta, \partial \Delta, \mathcal{U})} \Gamma(\Delta, \partial \Delta, \mathcal{U}) \\
& B_{\gamma}\left(\alpha_{1} \otimes \cdots \otimes \alpha_{n}\right)\left(f_{1}, \ldots, f_{m}\right)=\sum_{(\Delta, \partial \Delta) \in D(P)} C_{\Delta, \partial \Delta}\left(\alpha_{1} \otimes \cdots \otimes \alpha_{n}\right) \\
& \sum_{\mathcal{U}} b_{(\Delta, \partial \Delta, \mathcal{U})} \partial_{U_{1}} f_{1} \cdots \partial_{U_{m}} f_{m} .
\end{aligned}
$$

\section{Cohomologie de Hochschild}

Dans [1], on a traduit l'opérateur de cohomologie de Hochschild $d_{H}$ des opérateurs multidifférentiels (voir [3] par exemple) en un opérateur de cohomologie $d$ défini directement sur les graphes $\Gamma$ en posant :

$$
\begin{aligned}
& d\left(\Gamma_{\mathcal{U}}^{\Delta, \partial \Delta}\right)=d\left(\Gamma_{\left(U_{1}, \ldots, U_{m}\right)}^{\Delta, \partial \Delta}\right) \\
& =\Gamma_{\left(\varnothing, U_{1}, \ldots, U_{m}\right)}^{\Delta, \partial \Delta}+\sum_{\ell=1}^{m}(-1)^{\ell} \sum_{V^{\prime} \sqcup V^{\prime \prime}=U_{\ell}} \Gamma_{\left(U_{1}, \ldots, V^{\prime}, V^{\prime \prime}, \ldots, U_{m}\right)}^{\Delta, \partial \Delta} \\
& +(-1)^{m+1} \Gamma_{\left(U_{1}, \ldots, U_{m}, \varnothing\right)}^{\Delta, \partial \Delta} \\
& =\sum_{\substack{V^{\prime} \sqcup V^{\prime \prime}=U_{\ell} \neq \varnothing \\
V^{\prime}, V^{\prime \prime} \neq \varnothing}}(-1)^{\ell} \Gamma_{\left(U_{1}, \ldots, V^{\prime}, V^{\prime \prime}, \ldots, U_{m}\right)}^{\Delta, \partial \Delta}+\sum_{U_{\ell}=\varnothing}(-1)^{\ell+1} \Gamma_{\left(U_{1}, \ldots, \varnothing, \varnothing, \ldots, U_{m}\right)}^{\Delta, \partial \Delta} .
\end{aligned}
$$


Proposition (cohomologie des graphes, [1]). - Avec nos notations, on a pour tout $\alpha_{1} \otimes \cdots \otimes \alpha_{n}$ :

$$
B_{d \gamma}\left(\alpha_{1} \otimes \cdots \otimes \alpha_{n}\right)=d_{H} B_{\gamma}\left(\alpha_{1} \otimes \cdots \otimes \alpha_{n}\right)
$$

\section{Présentation algébrique de la cohomologie}

Dans [4], Kontsevich donne une formulation purement algébrique de l'opérateur de cohomologie de Hochschild en termes de la comultiplication d'une cogèbre libre. Il est facile de traduire cela directement sur les graphes. Fixons un graphe aérien $\Delta$ ayant $n$ sommets. Appelons $W_{\Delta}$ l'espace vectoriel de dimension $n$ engendré par les graphes $\Gamma$ ayant un seul pied et une seule patte et tels que $\Delta(\Gamma)=\Delta$. Une base de $W_{\Delta}$ est formée par les graphes $\Gamma_{i}$ dont la patte est

$$
\overrightarrow{p_{i} q_{1}}\left(\Gamma_{i}=\Gamma\left(\Delta,\left\{\left(p_{i}, 1\right)\right\},\left\{p_{i}\right\}\right)=\Gamma_{\left(\left\{p_{i}\right\}\right)}^{\Delta,\left\{\left(p_{i}, 1\right)\right\}}\right) .
$$

À partir de l'espace $W_{\Delta}$, on construit une algèbre $\mathcal{C}\left(W_{\Delta}\right)$ de la façon suivante :

- l'espace sous-jacent à $\mathcal{C}\left(W_{\Delta}\right)$ est l'espace $\wedge W_{\Delta}$ de base $\Gamma_{U}$ où $U$ est une partie de $\{1, \ldots, n\}$ (ou de $\left\{p_{1}, \ldots, p_{n}\right\}, \Gamma_{U}$ est le graphe à un seul pied $\Gamma_{\{U\}}^{\Delta, U \times\{1\}}$

- la multiplication, notée $\wedge$ est définie par

$$
\Gamma_{U} \wedge \Gamma_{U^{\prime}}= \begin{cases}\varepsilon\left(U, U^{\prime}\right) \Gamma_{U \cup U^{\prime}} & \text { si } U \cap U^{\prime}=\varnothing \\ 0 & \text { sinon, }\end{cases}
$$

où $\varepsilon\left(U, U^{\prime}\right)$ est la signature de la permutation rangement de $U \cup U^{\prime}$ qui consiste à remettre les indices de $U$ et de $U^{\prime}$ dans l'ordre croissant.

Par construction, en identifiant $\Gamma_{\left\{i_{1}, \ldots, i_{r}\right\}}$ à $\Gamma_{i_{1}} \wedge \cdots \wedge \Gamma_{i_{r}}$, on définit ainsi un isomorphisme entre $\mathcal{C}\left(W_{\Delta}\right)$ et l'algèbre $\wedge W_{\Delta}$. Rappelons d'ailleurs que les algèbres symétriques et extérieures sont deux avatars des algèbres symétriques sur un espace gradué. Enfin il est clair qu'avec nos notations,

$$
B_{\Gamma_{U} \wedge \Gamma_{U^{\prime}}}\left(\alpha_{1} \otimes \cdots \otimes \alpha_{n}\right)(f)=B_{\Gamma_{U \cup U^{\prime}}}\left(\alpha_{1} \otimes \cdots \otimes \alpha_{n}\right)(f) .
$$

Maintenant, suivant l'idée de Kontsevich, on note $S(\mathcal{C})$ la cogèbre libre coengendrée par $\mathcal{C}\left(W_{\Delta}\right)$. Un élément de $S(\mathcal{C})$ est donc une combinaison linéaire de produits tensoriels $\Gamma_{U_{1}} \otimes \cdots \otimes \Gamma_{U_{m}}$. On identifiera ce produit au graphe

$$
\Phi\left(\Gamma_{U_{1}} \otimes \cdots \otimes \Gamma_{U_{m}}\right)=\Gamma_{\left(U_{1}, \ldots, U_{m}\right)}=\Gamma_{\left(U_{1}, \ldots, U_{m}\right)}^{\Delta, \partial_{\left(U_{1}, \ldots, U_{m}\right)} \Delta} .
$$

Ainsi $S(\mathcal{C})$ est identifié par $\Phi$ à l'espace $V_{P}^{\Delta}$ de tous les graphes $\Gamma$ tels que $\Delta(\Gamma)=\Delta$. 
Associé à la comultiplication coassociative de $S(\mathcal{C})$ :

$$
\begin{aligned}
& \Delta: S(\mathcal{C}) \longrightarrow S(\mathcal{C}) \otimes S(\mathcal{C}), \\
& \Delta\left(\Gamma_{U_{1}} \otimes \cdots \otimes \Gamma_{U_{m}}\right)=\sum_{\ell=1}^{m-1} \Gamma_{U_{1}} \otimes \cdots \otimes \Gamma_{U_{\ell}} \otimes \Gamma_{U_{\ell+1}} \otimes \cdots \otimes \Gamma_{U_{m}},
\end{aligned}
$$

l'opérateur de cohomologie « algébrique » $d_{\text {alg }}$ est

$d_{\mathrm{alg}}: \bigotimes^{m} \mathcal{C} \longrightarrow \bigotimes^{m+1} \mathcal{c}$

$d_{\text {alg }}=\Gamma_{\varnothing} \otimes \operatorname{id}_{\otimes^{m} \mathcal{C}}+\sum_{\ell=1}^{m}(-1)^{\ell} \mathrm{id} \otimes \cdots \otimes \Delta_{\ell} \otimes \cdots \otimes \mathrm{id}+(-1)^{m+1} \operatorname{id}_{\otimes^{m} \mathcal{C}} \otimes \Gamma_{\varnothing}$.

( $\Delta_{\ell}$ est l'opérateur $\Delta$ appliqué au facteur numéro $\ell$.)

Proposition ( $d$ est l'image de $d_{\text {alg }}$ par $\left.\Phi\right)$. - Avec les notations ci-dessus :

$$
d=\Phi \circ d_{\text {alg }} \circ \Phi^{-1} \text {. }
$$

Corollaire ( $d$ est un opérateur de cohomologie). - L'opérateur $d_{\text {alg }}$ est une codérivation de degré 1 de $S(\mathcal{C})$, la coassociativité de $\Delta$ implique $d_{\mathrm{alg}} \circ d_{\mathrm{alg}}=0$. L'opérateur d est un opérateur de cohomologie sur $V_{P}^{\Delta}$.

En effet, si $C=\Gamma_{U_{1}} \otimes \cdots \otimes \Gamma_{U_{m}}$ et $C^{\prime}=\Gamma_{V_{1}} \otimes \cdots \otimes \Gamma_{V_{m^{\prime}}}$, on a par construction

$$
d_{\mathrm{alg}}\left(C \otimes C^{\prime}\right)=d_{\mathrm{alg}} C \otimes C^{\prime}+(-1)^{1 \cdot m} C \otimes d_{\mathrm{alg}} C^{\prime}
$$

ce que l'on note comme dans [2] :

$$
\Delta \circ d_{\text {alg }}=(\mathrm{id}+\tau)\left(d_{\text {alg }} \otimes \mathrm{id}\right) \circ \Delta .
$$

Pour prouver que $d \circ d=0$, ou ce qui revient au même que $d_{\text {alg }} \circ d_{\text {alg }}=0$, il nous suffit de considérer cet opération sur les générateurs $\Gamma_{U}$ de $\mathcal{C}$. Mais alors :

$$
\begin{aligned}
d_{\mathrm{alg}} \Gamma_{U} & =\Gamma_{\varnothing} \otimes \Gamma_{U}-\Delta \Gamma_{U}+\Gamma_{U} \otimes \Gamma_{\varnothing}, \\
\left(d_{\mathrm{alg}} \circ d_{\mathrm{alg}}\right) \Gamma_{U} & =\Gamma_{\varnothing} \otimes \Gamma_{\varnothing} \otimes \Gamma_{U}-\Delta \Gamma_{\varnothing} \otimes \Gamma_{U}+\Gamma_{\varnothing} \otimes \Delta \Gamma_{U}-\Gamma_{\varnothing} \otimes \Gamma_{U} \otimes \Gamma_{\varnothing} \\
& -\Gamma_{\varnothing} \otimes \Delta \Gamma_{u}+(\Delta \otimes i d) \circ \Delta \Gamma_{U}-(i d \otimes \Delta) \circ \Delta \Gamma_{U}+\Delta \Gamma_{U} \otimes \Gamma_{\varnothing} \\
& +\Gamma_{\varnothing} \otimes \Gamma_{U} \otimes \Gamma_{\varnothing}-\Delta \Gamma_{U} \otimes \Gamma_{\varnothing}+\Gamma_{U} \otimes \Delta \Gamma_{\varnothing}-\Gamma_{U} \otimes \Gamma_{\varnothing} \otimes \Gamma_{\varnothing} .
\end{aligned}
$$

Et de remarquer que $\Delta \Gamma_{\varnothing}=\Gamma_{\varnothing} \otimes \Gamma_{\varnothing}$.

\section{Réduction aux vrais graphes}

DÉfinition (graphes boîteux et vrais graphes). - On dira que le graphe $\Gamma_{\mathcal{U}}=\Gamma_{\left(U_{1}, \ldots, U_{m}\right)}$ est boîteux si au moins l'un des $U_{\ell}$ est vide; si au contraire tous les $U_{\ell}$ sont non vides, on dira que $\Gamma_{\mathcal{U}}$ est un vrai graphe. On notera $X$ l'espace engendré par les graphes boîteux et $Y$ celui des vrais graphes. $X^{\Delta}$ (resp. $Y^{\Delta}$ ) représentera l'espace des graphes $\Gamma$ boîteux (resp. vrais) tels que $\Delta(\Gamma)=\Delta$. 
Comme le remarque Kontsevich dans [4], on peut se restreindre à l'espace $Y$. En effet, si $\Delta$ est fixé, on vérifie immédiatement que

$$
V^{\Delta}=X^{\Delta} \oplus Y^{\Delta}, \quad d\left(Y^{\Delta}\right) \subset Y^{\Delta}, \quad d\left(X^{\Delta}\right) \subset X^{\Delta}
$$

et donc

$$
H^{\bullet}\left(V^{\Delta}\right)=H^{\bullet}\left(X^{\Delta}\right) \oplus H^{\bullet}\left(Y^{\Delta}\right) .
$$

Proposition (réduction aux vrais graphes). — La cohomologie des graphes bôtteux est nulle :

$$
H^{\bullet}\left(X^{\Delta}\right)=0 .
$$

Démonstration. - En suivant la présentation de Berger dans [3] et en revenant à l'expression algébrique de $d$, on remarque que

$$
d_{\mathrm{alg}} \Gamma_{U}=\sum_{V \sqcup V^{\prime}=U} \Gamma_{V} \otimes \Gamma_{V^{\prime}} \quad \text { si } \quad U \neq \varnothing
$$

et

$$
d_{\text {alg }}\left(\otimes^{m} \Gamma_{\varnothing}\right)=d_{\text {alg }}\left(\Gamma_{\varnothing} \otimes \cdots \otimes \Gamma_{\varnothing}\right)= \begin{cases}0 & \text { si } m \text { est pair, } \\ \otimes^{m+1} \Gamma_{\varnothing} & \text { si } m \text { est impair. }\end{cases}
$$

Donc si on représente nos combinaisons linéaires $\gamma$ de graphes par leur image dans $S(\mathcal{C})$ :

$$
\begin{aligned}
\gamma & =\sum_{U_{1} \neq \varnothing} a_{\left(U_{1}, \ldots, U_{m}\right)} \Gamma_{U_{1}} \otimes \cdots \otimes \Gamma_{U_{m}}+\sum_{U_{1}=\varnothing} a_{\left(\varnothing, U_{2}, \ldots, U_{m}\right)} \Gamma_{\varnothing} \otimes \cdots \otimes \Gamma_{U_{m}} \\
& =\gamma_{1}+\gamma_{>1},
\end{aligned}
$$

alors si $\gamma$ est un cocycle :

$$
\begin{aligned}
0= & d_{\mathrm{alg}} \gamma=d_{\mathrm{alg}} \gamma_{1}+\sum_{U_{1}=\varnothing} a_{\left(\varnothing, U_{2}, \ldots, U_{m}\right)} \\
& \left(\Gamma_{\varnothing} \otimes \Gamma_{\varnothing} \otimes \cdots \otimes \Gamma_{U_{m}}-\Gamma_{\varnothing} \otimes d_{\mathrm{alg}}\left(\Gamma_{U_{2}} \otimes \cdots \otimes \Gamma_{U_{m}}\right)\right) \\
= & \sum_{\mathcal{V}} b_{\left(V_{1}, \ldots, V_{m+1}\right)} \Gamma_{V_{1}} \otimes \cdots \otimes \Gamma_{V_{m+1}} .
\end{aligned}
$$

En particulier :

$$
0=b_{\left(\varnothing, \varnothing, V_{3}, \ldots, V_{m+1}\right)}=a_{\left(\varnothing, V_{3}, \ldots, V_{m+1}\right)}+\sum_{\ell=3}^{m}(-1)^{\ell} a_{\left(\varnothing, \varnothing, V_{3}, \ldots, V_{\ell} \cup V_{\ell+1}, \ldots, V_{m+1}\right)} .
$$

On en déduit que si

$$
\beta_{1}=\sum_{U_{3}, \ldots, U_{m}} a_{\left(\varnothing, \varnothing, U_{3}, \ldots, U_{m}\right)} \Gamma_{\varnothing} \otimes \Gamma_{U_{3}} \otimes \cdots \otimes \Gamma_{U_{m}},
$$

alors $d_{\mathrm{alg}} \beta_{1}=\gamma_{>1}$ et, modulo un cobord, on peut supposer que $\gamma$ se réduit à $\gamma_{1}$.

BULLETIN DE LA SOCiÉTÉ MATHÉMATIQUe DE FRANCE 
Par induction, on suppose maintenant que $\gamma$ est une combinaison linéaire de termes de la forme $\Gamma_{U_{1}} \otimes \cdots \otimes \Gamma_{U_{m}}$ avec $U_{1}, \ldots, U_{r-1}$ non vides. On écrit de nouveau

$$
\gamma=\gamma_{r}+\gamma_{>r}
$$

en regroupant dans $\gamma_{r}$ les termes $\Gamma_{\mathcal{U}}$ tels que $U_{r} \neq \varnothing$ et dans $\gamma_{>r}$ ceux pour lesquels $U_{r}=\varnothing$. Le même calcul, donne dans $d_{\text {alg }} \gamma$ :

$$
\begin{aligned}
0 & =b_{\left(V_{1}, \ldots, V_{r-1}, \varnothing, \varnothing, V_{r+2}, \ldots, V_{m+1}\right)} \\
& =(-1)^{r-1} a_{\left(V_{1}, \ldots, V_{r-1}, \varnothing, V_{r+2}, \ldots, V_{m+1}\right)} \\
& \sum_{\ell=r+2}^{m}(-1)^{\ell} a_{\left(V_{1}, \ldots, V_{r-1}, \varnothing, \varnothing, V_{r+2}, \ldots, V_{\ell} \cup V_{\ell+1}, \ldots, V_{m+1}\right) .}
\end{aligned}
$$

On en déduit que si

$$
\begin{aligned}
& \beta_{r}=\sum_{\substack{U_{1}, \ldots, U_{r-1}, U_{r+2}, \ldots, U_{m}}} a_{\left(U_{1}, \ldots, U_{r-1}, \varnothing, \varnothing, U_{r+2}, \ldots, U_{m}\right)} \\
& \Gamma_{U_{1}} \otimes \cdots \otimes \Gamma_{U_{r-1}} \otimes \Gamma_{\varnothing} \otimes \Gamma_{U_{r+2}} \otimes \cdots \otimes \Gamma_{U_{m}},
\end{aligned}
$$

alors $d_{\mathrm{alg}} \beta_{r}=(-1)^{r-1} \gamma_{>r}$ et, modulo un cobord, on peut supposer que $\gamma$ se réduit à $\gamma_{r}$. Si $\gamma$ est boîteux, tous les cobords introduits le sont aussi donc $\gamma_{m+1}=0, \gamma$ est un cobord.

À partir de maintenant, on ne considère que des vrais graphes. Pour ces graphes, si $k$ est le cardinal de $\partial \Delta$, on a $k \geq m$. Notons $Y^{(k), m}$ l'espace des vrais graphes ayant $m$ pieds et tels que le cardinal de $\partial \Delta$ est $k$. On a $d\left(Y^{(k), m}\right) \subset$ $Y^{(k), m+1}$, la cohomologie se restreint aux espaces $Y^{(k)}=\bigoplus_{m} Y^{(k), m}$.

\section{Le cas $k=m$}

Définissons d'abord l'action du groupe $\mathfrak{S}_{m}$ sur un graphe $\Gamma$ à $m$ pieds. Si $\sigma$ est dans $\mathfrak{S}_{m}$, on peut le faire agir sur $\Gamma$ en posant :

$$
\Gamma_{\left(U_{1}, \ldots, U_{m}\right)}^{\sigma}=\Gamma_{\left(U_{\sigma(1)}, \ldots, U_{\sigma(m))}\right.} .
$$

Mais il faut aussi tenir compte de l'orientation des graphes : si on ne change pas l'ordre des flèches arrivant sur $q_{1}, \ldots, q_{m}$, on obtient une orientation $\left(\mathcal{O}_{0}\right)^{\sigma}$ distincte de l'orientation canonique $\left(\mathcal{O}^{\sigma}\right)_{0}$. On posera donc :

$$
\sigma(\Gamma)=\varepsilon\left(\left(\mathcal{O}_{0}\right)^{\sigma},\left(\mathcal{O}^{\sigma}\right)_{0}\right) \Gamma^{\sigma} .
$$

(C'est encore une action de $\mathfrak{S}_{m}$ puisque c'est le produit tensoriel de l'action sur les graphes par celle sur les ordres.) Remarquons que

$$
\varepsilon(\sigma) \varepsilon\left(\left(\mathcal{O}_{0}\right)^{\sigma},\left(\mathcal{O}^{\sigma}\right)_{0}\right)=\varepsilon\left(\left(\mathcal{O}_{0}\right)^{\sigma}, \mathcal{O}_{0}\right)
$$

si $\varepsilon(\sigma)$ est la signature de $\sigma$.

TOME $130-2002-\mathrm{N}^{\mathrm{O}} 1$ 
DÉfinition (antisymétrisé). — L'antisymétrisé $a(\Gamma)$ du graphe $\Gamma$ est par définition

$$
a(\Gamma)=\frac{1}{m !} \sum_{\sigma \in \mathfrak{S}_{m}} \varepsilon(\sigma) \sigma(\Gamma)
$$

$(\varepsilon(\sigma)$ est la signature de $\sigma)$. On étend par linéarité l'opérateur $a$ aux éléments $\gamma$ de $V_{P, Q}$.

On a comme dans [3] :

Proposition (réduction à $a(\gamma)$ ). — Soit $\gamma$ un élément de $Y^{(m), m}$, alors :

1) $\gamma$ est un cocycle $: d \gamma=0$;

2) $\gamma-a(\gamma)$ est un cobord : $\gamma=a(\gamma)+d \beta$;

3) $a(\gamma)$ est un cobord si et seulement si $a(\gamma)=0$.

Démonstration. - Rappelons qu'on a noté $\Gamma_{i}$ le graphe $\Gamma\left(\Delta,\left(\left\{p_{i}, 1\right\}\right),\left\{p_{i}\right\}\right)$. On a $d_{\mathrm{alg}} \Gamma_{i}=0$ par construction. Comme $d_{\mathrm{alg}}$ est une codérivation de $S(\mathcal{C})$ et $Y^{(m), m}$ est engendré par les graphes $\Phi\left(\Gamma_{i_{1}} \otimes \cdots \otimes \Gamma_{i_{m}}\right)$, tout élément de $Y^{(m), m}$ coborde.

Si $p_{i_{\ell}} \neq p_{i_{\ell+1}}$, on a :

$$
\begin{aligned}
d_{\mathrm{alg}}\left(\Gamma_{i_{1}} \otimes\right. & \left.\cdots \otimes \Gamma_{i_{l-1}} \otimes \Gamma_{\left\{p_{i_{\ell}}, p_{i_{\ell+1}}\right\}} \otimes \Gamma_{i_{\ell+2}} \otimes \cdots \otimes \Gamma_{i_{m}}\right) \\
& =(-1)^{\ell}\left(\Gamma_{i_{1}} \otimes . . \otimes \Gamma_{i_{m}}+\Gamma_{i_{1}} \otimes \cdots \otimes \Gamma_{i_{\ell+1}} \otimes \Gamma_{i_{\ell}} \otimes \cdots \otimes \Gamma_{i_{m}}\right)
\end{aligned}
$$

et $\varepsilon\left(\left(\mathcal{O}_{0}\right)^{(\ell, \ell+1)},\left(\mathcal{O}^{(\ell, \ell+1)}\right)_{0}\right)=1$. Soit

$$
\Gamma_{i_{1}} \otimes \cdots \otimes \Gamma_{i_{m}}=\varepsilon((\ell, \ell+1))(\ell, \ell+1)\left(\Gamma_{i_{1}} \otimes \cdots \otimes \Gamma_{i_{m}}\right)+d \beta .
$$

D'autre part, si $p_{i_{\ell}}=p_{i_{\ell+1}}$, on a $\varepsilon\left(\left(\mathcal{O}_{0}\right)^{(\ell, \ell+1)},\left(\mathcal{O}^{(\ell, \ell+1)}\right)_{0}\right)=-1$ et donc

$$
\Gamma_{i_{1}} \otimes \cdots \otimes \Gamma_{i_{m}}=\varepsilon((\ell, \ell+1))(\ell, \ell+1)\left(\Gamma_{i_{1}} \otimes \cdots \otimes \Gamma_{i_{m}}\right) .
$$

Ceci prouve le point 2).

Si $a(\gamma)=d \beta$, chaque $\Gamma$ qui apparaît dans $\beta$ appartient à $Y^{(m), m-1}$, il a donc un pied à deux pattes et un seul : $\beta=\sum_{\ell=1}^{m-1} \beta_{\ell}$, où $\beta_{\ell}$ est la somme des composantes $\Gamma$ ayant deux pattes sur le pied $\ell$. Mais le calcul fait ci-dessus montre que pour un tel $\Gamma,(\ell, \ell+1)(d \Gamma)=d \Gamma$. On a donc :

$$
a(d \Gamma)=0, \quad a\left(d \beta_{\ell}\right)=0, \quad a(a(\gamma))=0=a(\gamma)
$$

Ce qui prouve 3).

Corollaire $\left(H^{m}\left(Y^{(m), m}\right)=a\left(Y^{(m), m}\right)\right)$. - Le $m$-ième groupe de cohomologie de $Y^{(m), m}$ est l'espace $a\left(Y^{(m), m}\right)$. Plus précisément, pour chaque couple $(\Delta, \partial \Delta)$, la cohomologie de l'espace $Y^{(\Delta, \partial \Delta)}$ des vrais graphes de la forme $\Gamma(\Delta, \partial \Delta, \mathcal{U})$ vérifie :

$$
H^{m}\left(Y^{(\Delta, \partial \Delta)}\right)=0 \quad \text { si } \quad m>\# \partial \Delta, \quad \operatorname{dim} H^{\# \partial \Delta}\left(Y^{(\Delta, \partial \Delta)}\right)=1 .
$$

BULletin DE LA SOCIÉtÉ MATHÉMATIQUE DE FRANCE 


\section{La suite spectrale $E^{\bullet, \bullet}$}

Fixons $\Delta$ et $\partial \Delta$ tel que $\# \partial \Delta=k$ et notons simplement $Y$ l'espace $Y^{\Delta, \partial \Delta}$ gradué par le nombre $m$ des pieds des graphes. Pour chaque $\gamma$ de $Y$

$$
\gamma=\sum_{\left(U_{1}, \ldots, U_{m}\right)} a_{\left(U_{1}, \ldots, U_{m}\right)} \Gamma\left(\Delta, \partial \Delta,\left(U_{1}, \ldots, U_{m}\right)\right)=\sum_{\mathcal{U}} a_{\mathcal{U}} \Gamma_{\mathcal{U}}^{\Delta, \partial \Delta} \in \bigoplus_{m=1}^{k} Y^{m},
$$

on appelle

$$
o(\gamma)=\sup \left\{\# U_{1}, a_{\left(U_{1}, \ldots, U_{m}\right)} \neq 0\right\} \quad(o(0)=-\infty) .
$$

On note $Y_{p}=\bigoplus_{m} Y_{p}^{m}$ l'espace des $\gamma$ de $Y$ tels que $o(\gamma) \leq k-p$ (c'est-à-dire tels que pour tout graphe $\Gamma$ de $\gamma$, au moins $p$ pattes arrivent sur les pieds numéros $2, \ldots, m)$. On a :

$$
Y_{p}=0 \quad \text { si } \quad p \geq k, \quad Y_{0}=Y, \quad Y_{p+1} \subset Y_{p} .
$$

On définit ensuite les espaces suivants :

$$
\begin{aligned}
& Z_{r}^{p, q}=\left\{\gamma \in Y_{p}^{p+q} \text { tels que } d \gamma \in Y_{p+r}^{p+q+1}\right\} \\
& B_{r}^{p, q}=\left\{\gamma \in Y_{p}^{p+q} \text { tels qu'il existe } \beta \in Y_{p-r}^{p+q-1} \text { tel que } \gamma=d \beta\right\}, \\
& E_{r}^{p, q}=Z_{r}^{p, q} /\left(B_{r-1}^{p, q}+Z_{r-1}^{p+1, q-1}\right) .
\end{aligned}
$$

Il est facile de vérifier que $E_{r}^{p, q}$ est bien défini, c'est-à-dire que $B_{r-1}^{p, q}+$ $Z_{r-1}^{p+1, q-1}$ est un sous-espace de $Z_{r}^{p, q}$. De plus :

Lemme (comparaison des $\left.E_{r}^{p, q}\right)$. - L'espace $E_{r+1}^{p, q}$ est un sous-quotient de l'espace $E_{r}^{p, q}$. En particulier, si $E_{r}^{p, q}=0$, alors $E_{r+1}^{p, q}=0$.

Démonstration. - Soit $i$ l'injection canonique de $Z_{r+1}^{p, q}$ dans $Z_{r}^{p, q}$ déduite de celle de $Y_{p+r+1}^{p+q}$ dans $Y_{p+r}^{p+q}, \pi$ la surjection canonique de $Z_{r}^{p, q}$ sur $E_{r}^{p, q}$ et $f$ l'application $\pi \circ i$. Notons $A$ l'espace $f\left(Z_{r+1}^{p, q}\right), B$ l'espace $f\left(B_{r}^{p, q}+Z_{r}^{p+1, q-1}\right)$ et $\varphi$ l'application quotient de $E_{r+1}^{p, q}$ sur $A / B$.

$\mathrm{Si}[\gamma]$ est dans le noyau de $\varphi$, c'est-à-dire si $\gamma$ est un élément de $Z_{r+1}^{p, q}$ tel que $f(\gamma)$ appartient à $B$, on peut écrire

$$
\gamma=\gamma_{1}+d \beta_{1}+\gamma_{0}+d \beta_{0}
$$

avec, si $m=p+q$,

$$
\left\{\begin{array} { l } 
{ \gamma \in Y _ { p } ^ { m } , \quad d \gamma \in Y _ { p + r + 1 } ^ { m + 1 } , } \\
{ \gamma _ { 1 } \in Y _ { p + 1 } ^ { m } , d \gamma _ { 1 } \in Y _ { p + r + 1 } ^ { m + 1 } , } \\
{ \gamma _ { 0 } \in Y _ { p + 1 } ^ { m } , d \gamma _ { 0 } \in Y _ { p + r } ^ { m + 1 } , }
\end{array} \quad \text { et } \quad \left\{\begin{array}{l}
\beta_{1} \in Y_{p-r}^{m-1}, \quad d \beta_{1} \in Y_{p}^{m} \\
\beta_{0} \in Y_{p-r+1}^{m-1}, d \beta_{0} \in Y_{p}^{m}
\end{array}\right.\right.
$$

Mais alors $d \gamma_{0}=d \gamma-d \gamma_{1}$ est dans $Y_{p+r+1}^{m+1}, \gamma_{0}+\gamma_{1}$ dans $Z_{r}^{p+1, q-1}$; de même, $\beta_{0}+\beta_{1}$ appartient à $Y_{p-r}^{m-1}$ et $d \beta_{0}+d \beta_{1}$ à $Y_{p}^{m}$, donc à $B_{r}^{p, q}$. C'est-à-dire $[\gamma]=0$, $\varphi$ est une bijection.

TOME $130-2002-\mathrm{N}^{\mathrm{O}} 1$ 


\section{Résultat principal}

ThÉORÈme (nullité des $E_{2}^{p, q}$ ). - On a $E_{2}^{p, q}=0$ sauf si $p=k-1$ et $q=1$ (dans ce cas $m=p+q=k$ ).

Corollaire (nullité des $\left.H^{\bullet}\right)$. - On a $H^{m}\left(Y^{(\Delta, \partial \Delta)}\right)=0$ si $m<k=\# \partial \Delta$.

Démonstration. - Soit $\gamma$ un cocycle $\left(\gamma\right.$ appartient à $Y^{m}$ et $\left.d \gamma=0\right)$, soit $p$ le nombre minimal de pattes des graphes $\Gamma$ apparaissant dans $\gamma$ qui arrivent sur les pieds $q_{2}, \ldots, q_{m}(k>p \geq m-1)$, soit $q=m-p$. Alors $\gamma$ appartient à $Z_{2}^{p, q}$, comme $m=p+q<k, E_{2}^{p, q}=0$ et il existe $\gamma_{1}$ dans $Z_{1}^{p+1, q-1}$ et $d \beta_{1}$ dans $B_{1}^{p, q}$ tels que

$$
\gamma=\gamma_{1}+d \beta_{1}
$$

La classe de $\gamma$ dans $H^{m}(Y)$ est celle de $\gamma_{1}$, mais maintenant $\gamma_{1}$ appartient à $Y_{p+1}^{m}$, c'est-à-dire qu'il y a au plus $k-(p+1)$ pattes qui arrivent sur $q_{1}$. On répète cette opération $k-p$ fois et on construit un cocycle $\gamma_{k-p}$ dans la classe de $\gamma$ tel qu'il n'y a aucune patte arrivant sur $q_{1} ; \gamma_{k-p}$ est boîteux, donc nul, $H^{m}\left(Y^{(\Delta, \partial \Delta)}\right)=0$.

Démonstration du théorème. — On prouve ce résultat par récurrence sur $k$.

- Si $k=1, Y^{(1), m}=0$ sauf si $m=1$ et $\operatorname{dim} Y_{0}^{1}=1$. Donc $Y_{p}^{m}=0$ pour tout $p \geq 1$ et $Z_{2}^{p, q}=0$ sauf si $p=0$ et $q=1$. Le théorème est vrai si $k=1$.

- Supposons-le vrai pour tout $k^{\prime}<k$ et prouvons-le pour $k$.

Premier pas. - Si $q \neq 1$, alors $E_{1}^{p, q}=0$. Soit $\gamma$ appartenant à $Z_{1}^{p, q}$. Écrivons $\gamma$ sous la forme :

$$
\gamma=\sum_{\# U_{1}=k-p} a_{\mathcal{U}} \Gamma_{\mathcal{U}}+\sum_{\# U_{1}<k-p} a_{\mathcal{U}} \Gamma_{\mathcal{U}}=\gamma_{1}+\gamma_{0}
$$

Par construction, $\gamma_{1}$ est dans $Y_{p}^{m}$ et $\gamma_{0}$ dans $Y_{p+1}^{m}$ donc $d \gamma_{0}$ appartient à $Y_{p+1}^{m+1}$, $\gamma_{0}$ est dans $Z_{0}^{p+1, q-1}$, dans $E_{1}^{p, q},[\gamma]=\left[\gamma_{1}\right]$. Notons ce $\gamma_{1}$ ainsi :

$$
\gamma_{1}=\sum_{U_{1}} \sum_{\mathcal{V}=\left(U_{2}, \ldots, U_{m}\right)} a_{U_{1}, \mathcal{V}} \Phi\left(\Gamma_{U_{1}} \otimes \Phi^{-1}\left(\Gamma_{\mathcal{V}}\right)\right)=\sum_{U_{1}, \mathcal{V}} a_{U_{1}, \mathcal{V}} \Gamma_{U_{1}} \vee \Gamma_{\mathcal{V}}
$$

Si $q \geq 2$, on a $p=m-q \leq m-2<m-1$, chaque $\Gamma_{\mathcal{V}}$ est boîteux, ce qui est impossible, $\gamma_{1}=0$.

Supposons maintenant $q \leq 0$, c'est-à-dire $p=m-q \geq m>m-1$. Ou si on pose $\gamma_{U_{1}}=\sum_{\mathcal{V}} a_{U_{1}, \mathcal{V}} \Gamma \mathcal{V}$

$$
\gamma_{1}=\sum_{U_{1}} \Gamma_{U_{1}} \vee \gamma_{U_{1}}
$$

Un calcul direct donne :

$$
d \gamma_{1}=\sum_{U_{1}}\left(\left(d \Gamma_{U_{1}}\right) \vee \gamma_{U_{1}}-\Gamma_{U_{1}} \vee\left(d \gamma_{U_{1}}\right)\right)
$$

BULlETIN DE LA SOCIÉtÉ MATHÉMATIQUE DE FRANCE 
Mais $\left(d \Gamma_{U_{1}}\right) \vee \gamma_{U_{1}}$ est dans $Y_{p+1}^{m+1}$, donc $\sum_{U_{1}} \Gamma_{U_{1}} \vee\left(d \gamma_{U_{1}}\right)$ aussi, mais ceci n'est possible que si $d \gamma_{U_{1}}=0$ pour tout $U_{1}$. Chaque $\gamma_{U_{1}}$ est un $(m-1)$ cocycle, donc, grâce à l'hypothèse de récurrence et au corollaire et puisque $\# \partial \Delta\left(\Gamma_{\mathcal{V}}\right)=p>m-1$, est un cobord. On peut donc écrire $\gamma_{U_{1}}=d \beta_{U_{1}}$ et

$$
\gamma_{1}=\sum_{U_{1}} \Gamma_{U_{1}} \vee \gamma_{U_{1}}=\sum_{U_{1}}\left(\left(d \Gamma_{U_{1}}\right) \vee \beta_{U_{1}}\right)-\sum_{U_{1}} d\left(\Gamma_{U_{1}} \vee \beta_{U_{1}}\right)
$$

Par construction, le second terme est dans $B_{0}^{p, q}$. Modulo ce terme, il ne reste dans $E_{1}^{p, q}$ que le premier terme qui est dans $Y_{p+1}^{m}$, donc nul dans $E_{1}^{p, q}, E_{1}^{p, q}=0$.

Intermède. - Sur le calcul de $E_{2}^{m-1,1}$ si $m<k$ : soit $[\gamma]$ un élément de $E_{2}^{m-1,1}$, c'est-à-dire

$$
\begin{array}{r}
\gamma=\sum_{\# U=k-m+1} \sum_{\mathcal{V}} a_{U, \mathcal{V}} \Gamma_{U, \mathcal{V}}+\sum_{\# U=k-m} \sum_{\mathcal{W}} a_{U, \mathcal{W}} \Gamma_{U, \mathcal{W}} \\
+\sum_{\# U<k-m} \sum_{\mathcal{X}} a_{U, \mathcal{X}} \Gamma_{U, \mathcal{X}}=\gamma_{-1}+\gamma_{0}+\gamma_{1}
\end{array}
$$

avec $\gamma \in Y_{m-1}^{m}$ et $d \gamma \in Y_{m+1}^{m+1}$. Alors $\gamma_{1}$ appartient à $Y_{m+1}^{m}$ donc à $Y_{m}^{m}$ et $d \gamma_{1}$ à $Y_{m+1}^{m+1}$, donc $\gamma_{1}$ est dans $Z_{1}^{m-1,1}$ et on peut le négliger en passant au quotient. Maintenant $\gamma=\gamma_{-1}+\gamma_{0}$ et, dans $\gamma_{-1}$, les divers $\mathcal{V}$ sont de la forme

$$
\mathcal{V}=\left(\left\{p_{j_{2}}\right\}, \ldots,\left\{p_{j_{m}}\right\}\right)
$$

tandis que dans $\gamma_{0}$, les $\mathcal{W}$ sont de la forme

$$
\mathcal{W}=\left(\left\{p_{j_{1}}\right\}, \ldots,\left\{p_{j_{\ell}}, p_{j_{\ell+1}}\right\}, \ldots,\left\{p_{j_{m}}\right\}\right) .
$$

Second pas : une relation sur $\mathcal{V}$. — Fixons une suite $J$ de $m$ éléments de $\partial \Delta$, avec peut-être des répétitions :

$$
J=\left(p_{j_{1}}, \ldots, p_{j_{m}}\right) \quad \text { et } \quad\left\{p_{j_{1}}, \ldots, p_{j_{m}}\right\}=\left\{p_{i_{1}}, \ldots, p_{i_{1}}, \ldots, p_{i_{s}}, \ldots, p_{i_{s}}\right\}
$$

( $p_{i_{1}}$ apparaît $r_{i_{1}}$ fois, $\ldots, p_{i_{s}}$ apparaît $r_{i_{s}}$ fois).

Notons $U$ le complémentaire de $J$ dans $\partial \Delta$, avec nos notations,

$$
\begin{aligned}
\Phi^{-1}\left(\Gamma_{\left(U \cup\left\{p_{j_{1}}\right\},\left\{p_{j_{2}}\right\}, \ldots,\left\{p_{j_{m}}\right\}\right)}\right)= & \pm\left(\Gamma_{U} \wedge \Gamma_{j_{1}}\right) \otimes \Gamma_{j_{2}} \otimes \cdots \otimes \Gamma_{j_{m}}, \\
\Phi^{-1}\left(\Gamma_{\left(U,\left\{p_{j_{1}}\right\}, \ldots,\left\{p_{j_{\ell}}, p_{j_{\ell+1}}\right\}, \ldots,\left\{p_{j_{m}}\right\}\right)}\right)= & \pm \Gamma_{U} \otimes \\
& \Gamma_{j_{1}} \otimes \cdots \otimes\left(\Gamma_{j_{\ell}} \wedge \Gamma_{j_{\ell+1}}\right) \otimes \cdots \otimes \Gamma_{j_{m}} .
\end{aligned}
$$

On écrit donc :

$$
\begin{aligned}
\Phi^{-1}(\gamma)=\sum_{J} & \sum_{\sigma \in \mathfrak{S}_{r_{1}} \times \cdots \times \mathfrak{S}_{r_{s}} \backslash \mathfrak{S}_{\mathfrak{m}}}\left\{a_{\sigma}^{J}\left(\Gamma_{U} \wedge \Gamma_{j_{\sigma(1)}}\right) \otimes \Gamma_{j_{\sigma(2)}} \otimes \cdots \otimes \Gamma_{j_{\sigma(m)}}\right. \\
& \left.+\sum_{\ell} b_{\sigma, \ell}^{J} \Gamma_{U} \otimes \Gamma_{j_{\sigma(1)}} \otimes \cdots \otimes\left(\Gamma_{j_{\sigma(\ell)}} \wedge \Gamma_{j_{\sigma(l+1)}}\right) \otimes \cdots \otimes \Gamma_{j_{\sigma(m)}}\right\} .
\end{aligned}
$$

TOME $130-2002-\mathrm{N}^{\mathrm{O}} 1$ 
Cherchons dans $\Phi^{-1}(d \gamma)$ les termes en $\Gamma_{U} \otimes \Gamma_{j_{\sigma(1)}} \otimes \cdots \otimes \Gamma_{j_{\sigma(m)}}$. Ils ont pour coefficient

$$
c_{\sigma}^{J}=a_{\sigma}^{J}+\sum_{\ell}(-1)^{\ell}\left(b_{\sigma, \ell}^{J}+b_{(\ell, \ell+1) \circ \sigma, \ell}^{J}\right) .
$$

Puisque $d \gamma$ est dans $Y_{m+1}^{m+1}$, ces termes doivent disparaître : $c_{\sigma}^{J}=0$ pour tout $J$ et tout $\sigma$.

Posons alors :

$$
\beta_{\sigma}^{J}=\sum_{\ell} b_{\sigma, \ell}^{J} \Gamma_{j_{\sigma(1)}} \otimes \cdots \otimes\left(\Gamma_{j_{\sigma(\ell)}} \wedge \Gamma_{j_{\sigma(l+1)}}\right) \otimes \cdots \otimes \Gamma_{j_{\sigma(m)}} .
$$

Un calcul direct donne

$$
d \beta_{\sigma}^{J}=\sum_{\ell}(-1)^{\ell}\left(b_{\sigma, \ell}^{J}+b_{(\ell, \ell+1) \circ \sigma, \ell}^{J}\right) \Gamma_{j_{\sigma(1)}} \otimes \cdots \otimes \Gamma_{j_{\sigma(m)}} .
$$

Donc $a\left(d \beta_{\sigma}^{J}\right)=0$ puisque $d \beta_{\sigma}^{J}$ est un cobord. Soit :

$$
\sum_{\tau \in \mathfrak{S}_{m}} \sum_{\ell} \varepsilon^{\prime}(\tau)(-1)^{\ell}\left(b_{\sigma \circ \tau, \ell}^{J}+b_{(\ell, \ell+1) \circ \sigma \circ \tau, \ell}^{J}\right)=0,
$$

où $\varepsilon^{\prime}(\tau)$ est le signe $\varepsilon\left(\left[\left(\mathcal{O}^{\sigma}\right)_{0}\right]^{\tau},\left(\mathcal{O}^{\sigma}\right)_{0}\right)$.

On a donc montré

Lemme (relation sur les $\left.a_{\sigma}^{J}\right)$. - Pour tout $J$ et tout $\sigma$, on a :

$$
\sum_{\tau \in \mathfrak{S}_{m}} \varepsilon^{\prime}(\tau) a_{\sigma \circ \tau}^{J}=0
$$

Troisième pas : choix de $j_{1}$ et rangement des indices $j_{\ell}$. - Modulo une modification du terme $\gamma_{0}$, on peut ranger les indices $j_{\ell}$ de $J$ dans l'ordre croissant. En effet, si $j_{\ell}>j_{\ell+1}$ dans

$$
\Gamma_{\left(V,\left\{p_{j_{2}}\right\}, \ldots,\left\{p_{j_{m}}\right\}\right)}=\Phi\left(\Gamma_{V} \otimes \Gamma_{j_{2}} \otimes \cdots \otimes \Gamma_{j_{m}}\right),
$$

on pose

$$
\beta=\Phi\left(\Gamma_{V} \otimes \cdots \otimes\left(\Gamma_{j_{\ell}} \wedge \Gamma_{j_{\ell+1}}\right) \otimes \cdots \otimes \Gamma_{j_{m}}\right) \in Y_{m-1}^{m-1} .
$$

Alors

$$
d \beta=(-1)^{\ell} \Phi\left(\Gamma_{V} \otimes \cdots \otimes\left(\Gamma_{j_{\ell}} \otimes \Gamma_{j_{\ell}}+\Gamma_{j_{\ell+1}} \otimes \Gamma_{j_{\ell}}\right) \otimes \cdots \otimes \Gamma_{j_{m}}\right)+\gamma_{0}^{\prime}
$$

avec $\gamma_{0}^{\prime}$ élément de $Y_{m}^{m}$. Par construction $d \beta$ appartient à $Y_{m-1}^{m}$ donc à $Z_{1}^{m, 0}$. On peut l'ajouter à $\gamma$, on modifie ainsi le terme «principal » $\gamma_{-1}$ et le terme «secondaire» $\gamma_{0}$. Il est clair que par répétition de cette opération, on peut supposer les indices $j_{2}, \ldots, j_{m}$ tous rangés dans l'ordre croissant :

$\forall V, \forall\left(j_{2}, \ldots, j_{m}\right), a_{\left(V,\left\{p_{j_{2}}\right\}, \ldots,\left\{p_{j_{m}}\right\}\right)}=0 \quad$ si $\left(j_{2}, \ldots, j_{m}\right)$ n'est pas croissante.

Notons $i_{0}$ le plus petit des indices de $\partial \Delta$. Si $p_{i_{0}}$ n'appartient pas à $V$, alors $p_{i_{0}}=p_{j_{2}}$ et on pose :

$$
\beta=\Phi\left(\Gamma_{V \sqcup\left\{p_{i_{0}}\right\}} \otimes \Gamma_{j_{3}} \otimes \cdots \otimes \Gamma_{j_{m}}\right) \in Y_{m-2}^{m-1} \quad(\# V=k-m>0) .
$$

BULletin DE LA SOCiÉtÉ MATHÉMATIQUE DE FRANCE 
Alors :

$d \beta=\Phi\left(\Gamma_{V} \otimes \Gamma_{i_{0}} \otimes \Gamma_{j_{3}} \otimes \cdots \otimes \Gamma_{j_{m}}+\sum_{p_{x} \in V}\left(\Gamma_{V \backslash\left\{p_{x}\right\} \sqcup\left\{p_{i_{0}}\right\}} \otimes \Gamma_{x} \otimes \Gamma_{j_{3}} \otimes \cdots \otimes \Gamma_{j_{m}}\right)\right)$.

De nouveau, $d \beta$ est un élément de $Z_{1}^{m, 0}$ et l'ajout de ce terme modifie $\gamma_{-1}$ $\left(\operatorname{pas} \gamma_{0}\right)$. Dans $d \beta$ chaque terme à part le premier vérifie $i_{0}$ appartient à $V$. On peut donc maintenant supposer que :

$\forall V, \forall\left(j_{2}, \ldots, j_{m}\right)$, si $i_{0} \notin V$ ou si $\left(j_{2}, \ldots, j_{m}\right)$ n'est pas croissante,

$$
a_{\left(V,\left\{p_{j_{2}}\right\}, \ldots,\left\{p_{j m}\right\}\right)}=0 .
$$

Quatrième pas : conclusion. - Reprenons les notations du pas 2. Les seuls $J$ qui apparaissent sont les suites rangées commençant par $j_{1}=i_{0}$, donc :

$$
\forall J, \quad a_{\sigma}^{J} \neq 0 \text { implique } \sigma=[i d] \text { dans } \mathfrak{S}_{r_{1}} \times \cdots \times \mathfrak{S}_{r_{s}} \backslash \mathfrak{S}_{n} .
$$

La relation du lemme s'écrit :

$$
\sum_{\tau \in \mathfrak{S}_{r_{1}} \times \cdots \times \mathfrak{S}_{r_{s}}} \varepsilon^{\prime}(\tau) a_{\tau}^{J}=r_{1} ! \ldots r_{s} ! a_{[\mathrm{id}]}^{J}=0 .
$$

Donc $\gamma_{-1}$ est nul, $\gamma=\gamma_{0}$ appartient à $Y_{m}^{m}$ et $d \gamma$ appartient à $Y_{m+1}^{m+1}, \gamma$ est dans $Z_{1}^{m, 0}$, donc $[\gamma]=0$ et si $m<k, E_{2}^{m-1,1}=0$.

\section{Interprétation cohomologique de l'équation de formalité pour $\mathbb{R}^{\bullet}$}

Rappelons brièvement les structures de $L_{\infty}$-algèbre de $T\left(\mathbb{R}^{\bullet}\right)$ et $D\left(\mathbb{R}^{\bullet}\right)$ (voir [4] ou [2]). Si $\alpha_{1}$ est un $k_{1}$-tenseur et $\alpha_{2}$ un $k_{2}$-tenseur alors :

$$
Q_{1}\left(\alpha_{1}\right)=0 \quad \text { et } \quad Q_{2}\left(\alpha_{1} \cdot \alpha_{2}\right)=-(-1)^{\left(k_{1}-1\right) k_{2}}\left[\alpha_{2}, \alpha_{1}\right]_{S}
$$

où $[,]_{S}$ est le crochet de Schouten. Si $A_{1}$ est un $m_{1}$-opérateur différentiel et $A_{2}$ est un $m_{2}$-opérateur différentiel et si $[,]_{G}$ désigne le crochet de Gerstenhaber alors :

$$
Q_{1}^{\prime}\left(A_{1}\right)=-d_{H} A_{1} \quad \text { et } \quad Q_{2}^{\prime}\left(A_{1} \cdot A_{2}\right)=(-1)^{\left(m_{1}-1\right) m_{2}}\left[A_{1}, A_{2}\right]_{G} .
$$

On cherche les formalités $\mathcal{F}$ sur $\mathbb{R}^{\bullet}$ construites à partir de combinaisons linéaires de graphes appartenant aux espaces $V_{n, m}$. On impose :

$$
\mathcal{F}_{(1)}(\alpha)\left(f_{1}, \ldots, f_{m}\right)=\left\langle\alpha, d f_{1} \wedge \cdots \wedge d f_{m}\right\rangle,
$$

donc $\mathcal{F}_{(1)}=\sum_{m} B_{\Gamma(m)}$ où $\Gamma(m)$ est l'unique vrai graphe $\Gamma$ à $m$ pieds et un seul sommet aérien :

$$
\begin{array}{r}
\Gamma(m)=\Gamma\left(\left\{p_{1}\right\},\left\{p_{1}\right\} \times\{1, \ldots, m\},\left(\left\{p_{1}\right\}, \ldots,\left\{p_{1}\right\}\right)\right) \in Y^{\left\{p_{1}\right\},\left\{p_{1}\right\} \times\{1, \ldots, m\}} \\
\subset Y^{(m), m} .
\end{array}
$$

Alors $d \Gamma(m)=0$ pour chaque $m$ et donc $d_{H} \mathcal{F}_{(1)}=0$.

TOME $130-2002-\mathrm{N}^{\mathrm{O}} 1$ 
On cherche ensuite à construire $\mathcal{F}_{(2)}, \ldots, \mathcal{F}_{(n)}$ par induction sur $n$ en résolvant l'équation de formalité :

$$
\begin{aligned}
d_{H}\left(\mathcal{F}_{(n)}\left(\alpha_{1} \ldots \alpha_{n}\right)\right) & =-\frac{1}{2} \sum_{k \neq \ell} \varepsilon_{\alpha}(k \ell, 1 \ldots \widehat{k \ell} \ldots n) \\
& +\frac{1}{2} \sum_{I \sqcup J=\{1, \ldots, n\} I \neq, J \neq} \sum_{(n-1)}\left(Q_{2}\left(\alpha_{k} \cdot \alpha_{\ell}\right) \cdot \alpha_{1} \ldots \widehat{\alpha_{k} \alpha_{\ell}} \ldots \alpha_{n}\right)
\end{aligned}
$$

où $\varepsilon_{\alpha}(I, J)$ désigne la signature de l'effet sur les $\alpha_{i}$ impairs de la permutation battement associée à la partition $\{I, J\}$ de $\{1, \ldots, n\}$.

On note $\mathcal{F}_{(n), m}\left(\alpha_{1} \ldots \alpha_{n}\right)$ la composante de $\mathcal{F}_{(n)}\left(\alpha_{1} \ldots \alpha_{n}\right)$ dans l'espace des opérateurs $m$-différentiels. On impose de plus une condition d'homogénéité sur les $\mathcal{F}_{(n), m}$ dans le sens où on demande que pour $n$ et $m$ fixés le nombre total $K$ de dérivations (portant sur les $\alpha_{i}$ et les $f_{i}$ ) est constant. Comme pour $\mathcal{F}_{(1), m}$ ce nombre vaut $m=2 \times 1+m-2$, un raisonnement par récurrence nous montre que les graphes qui apparaissent dans $\mathcal{F}_{(n), m}$ doivent tous avoir $2 n+m-2$ arêtes. Plus précisément on a la définition suivante :

DÉfinition (formalité de Kontsevich). - Une formalié de Kontsevich est une suite de combinaisons linéaires de graphes $\left(\gamma_{n, m}\right)_{(n, m) \in \mathbb{N}^{*} \times \mathbb{N}}$ vérifiant :

i) pour tout $(n, m)$ dans $\mathbb{N}^{*} \times \mathbb{N}, \gamma_{n, m}$ est dans $V_{n, m}$ et $\gamma_{1, m}=\Gamma(m)$;

ii) si $\gamma_{n, m}=\sum b_{\Gamma} \Gamma$ alors pour tout $\Gamma$ tel que $b_{\Gamma} \neq 0$ on a

$$
\# \mathcal{A}(\Gamma)=2 n+m-2
$$

iii) si on pose $\mathcal{F}_{(n)}=\sum_{m \in \mathbb{N}} B_{\gamma_{n, m}}$ alors les $\mathcal{F}_{(n)}$ vérifient l'équation de formalité.

ThÉORÈme (le second membre est un cocycle). - Si on a trouvé $\mathcal{F}_{(1)}$, $\ldots, \mathcal{F}_{(n-1)}$, solutions de l'équation à l'ordre $1, \ldots, n-1$, alors le second membre de cette équation à l'ordre $n$ est une somme de m-cocycles de Hochschild.

Avant de commencer la preuve du théorème, posons :

$$
\begin{aligned}
\delta\left(\mathcal{F}_{(n), m}\right)\left(\alpha_{1} \ldots \alpha_{n}\right)=\sum_{k \neq \ell} \varepsilon_{\alpha}(k \ell, 1 \ldots \widehat{k \ell} \ldots n) \\
\mathcal{F}_{(n-1), m}\left(Q_{2}\left(\alpha_{k} \cdot \alpha_{\ell}\right) \cdot \alpha_{1} \ldots \widehat{\alpha_{k} \alpha_{\ell}} \ldots \alpha_{n}\right) .
\end{aligned}
$$

On a alors le lemme suivant :

LEMme ( $\delta$ est un opérateur de cohomologie). - L'opérateur $\delta$ est un opérateur de cohomologie sur les applications multilinéaires de $T\left(\mathbb{R}^{\bullet}\right)$ dans $D\left(\mathbb{R}^{\bullet}\right)$ :

$$
\delta \circ \delta=0 .
$$

BULLETIN DE LA SOCIÉTÉ MATHÉMATIQUe DE FRANCE 
Démonstration du lemme. — Calculons directement :

$$
\begin{aligned}
& \delta\left(\delta \mathcal{F}_{(n-1), m}\right)\left(\alpha_{1} \ldots \alpha_{n+1}\right) \\
&= \sum_{\substack{\{i, j\} \neq\{k, \ell\} \\
i \neq j, k \neq \ell}} \varepsilon_{\alpha}(k \ell, 1 \ldots \widehat{k \ell} \ldots n+1) \varepsilon_{\tilde{\alpha}}(i j, 0,1 \ldots \widehat{k \ell i j} \ldots n+1) \\
&+ \sum_{\substack{j \neq k \ell, k \neq \ell \\
\varepsilon_{\alpha}}} \mathcal{F}_{(n-1), m}\left(Q_{2}\left(\alpha_{i} \cdot \alpha_{j}\right) \cdot Q_{2}\left(\alpha_{k} \cdot \alpha_{\ell}\right) \cdot \alpha_{1} \ldots \alpha_{k} \widehat{\alpha_{\ell} \alpha_{i}} \alpha_{j} \ldots \alpha_{n+1}\right) \\
&\left.\left.+\sum_{j \neq k \ell, k \neq \ell} \varepsilon_{\alpha}\left(k \ell, 1 \ldots \widehat{k \ell} \ldots \alpha_{k} \cdot \alpha_{\ell}\right) \cdot \alpha_{j}\right) \cdot \alpha_{1} \ldots \widehat{\alpha_{k} \alpha_{\ell} \alpha_{j}} \ldots \alpha_{n+1}\right) \\
& \mathcal{F}_{(n-1), m}\left(Q_{2}\left(\alpha_{j} \cdot Q_{2}\left(\alpha_{k} \cdot \alpha_{\ell}\right)\right) \cdot \alpha_{1} \ldots \widehat{\alpha_{k} \alpha_{\ell} \alpha_{j}} \ldots \alpha_{n+1}\right)
\end{aligned}
$$

où on a posé $\widetilde{\alpha}=\alpha_{0} \cdot \alpha_{1} \ldots \widehat{\alpha_{k} \alpha_{\ell}} \ldots \alpha_{n}$ et $\alpha_{0}=Q_{2}\left(\alpha_{k} \cdot \alpha_{\ell}\right)$. Les termes de la première somme s'annulent deux à deux. En effet,

$$
\begin{aligned}
\varepsilon_{\alpha}(k \ell, 1 \ldots \widehat{k \ell} \ldots n+1) \varepsilon_{\tilde{\alpha}}(i j, 0,1 \ldots \widehat{k \ell i j} \ldots n+1) \\
=(-1)^{\left(k_{k}+k_{\ell}-1\right)\left(k_{i}+k_{j}\right)} \varepsilon_{\tilde{\alpha}}(k \ell i j, 0,1 \ldots \widehat{k \ell i j} \ldots n+1)
\end{aligned}
$$

où $k_{s}$ est l'ordre du tenseur $\alpha_{s}$, par suite

$$
\begin{aligned}
& \varepsilon_{\alpha}(k \ell, 1 \ldots \widehat{k \ell} \ldots n+1) \varepsilon_{\tilde{\alpha}}(i j, 0,1 \ldots \widehat{k \ell i j} \ldots n+1) \\
& \mathcal{F}_{(n-1), m}\left(Q_{2}\left(\alpha_{i} \cdot \alpha_{j}\right) \cdot Q_{2}\left(\alpha_{k} \cdot \alpha_{\ell}\right) \cdot \alpha_{1} \ldots \alpha_{k} \widehat{\alpha_{\ell} \alpha_{i}} \alpha_{j} \ldots \alpha_{n+1}\right) \\
& =-\varepsilon_{\alpha}(i j, 1 \ldots \widehat{i j} \ldots n+1) \varepsilon_{\tilde{\alpha}}(k \ell, 0,1 \ldots \widehat{i j k \ell} \ldots n+1) \\
& \mathcal{F}_{(n-1), m}\left(Q_{2}\left(\alpha_{k} \cdot \alpha_{\ell}\right) \cdot Q_{2}\left(\alpha_{i} \cdot \alpha_{j}\right) \cdot \alpha_{1} \ldots \alpha_{i} \widehat{\alpha_{j} \alpha_{k}} \alpha_{\ell} \ldots \alpha_{n+1}\right) .
\end{aligned}
$$

La deuxième somme est nulle car $Q_{2}$ vérifie l'équation de Jacobi graduée (voir [4] ou [2]). En effet, d'une part

$$
\varepsilon_{\alpha}(k \ell, 1 \ldots \widehat{k \ell} \ldots n+1) \varepsilon_{\tilde{\alpha}}(0 j, 1 \ldots \widehat{k \ell j} \ldots n+1)=\varepsilon_{\alpha}(k \ell j, 1 \ldots \widehat{k \ell j} \ldots n+1)
$$

et d'autre part

$$
\begin{aligned}
& 0=\varepsilon_{\alpha}(k \ell j, 1 \ldots \widehat{k \ell j} \ldots n+1) \\
& \left(Q_{2}\left(Q_{2}\left(\alpha_{k} \cdot \alpha_{\ell}\right) \alpha_{j}\right)+(-1)^{k_{k}\left(k_{\ell}+k_{j}\right)} Q_{2}\left(Q_{2}\left(\alpha_{\ell} \cdot \alpha_{j}\right) \alpha_{k}\right)\right. \\
& \left.+(-1)^{k_{j}\left(k_{k}+k_{\ell}\right)} Q_{2}\left(Q_{2}\left(\alpha_{j} \cdot \alpha_{k}\right) \alpha_{\ell}\right)\right)
\end{aligned}
$$

Pour les mêmes raisons la troisième somme est nulle.

Remarque. - On a vu que l'opérateur $d_{H}$ se traduit par un opérateur $d$ sur les graphes de Kontsevich correspondant à l'« éclatement » d'un sommet terrestre en deux sommets terrestres. De même l'opérateur $\delta$ se traduit par un 
opérateur $\tilde{\delta}$ sur les graphes de Kontsevich correspondant à l'«éclatement » d'un sommet aérien en deux sommets aériens avec l'ajout d'une arête qui les relie (dans un sens ou l'autre).

Démonstration du théorème. — Pour tout $I \subset\{1, \ldots, n\}$ on pose $m_{I}=|I|-$ $2 n+2$. Pour alléger les écritures tous les ensembles qui apparaissent sous le signe $\sum$ sont supposés non vides. Le second membre de l'équation de formalité à l'ordre $n$ est une somme de termes de la forme :

$$
\begin{aligned}
& -\frac{1}{2} \sum_{k \neq \ell} \varepsilon_{\alpha}(k \ell, 1 \ldots \widehat{k \ell} \ldots n) \mathcal{F}_{(n-1), m}\left(Q_{2}\left(\alpha_{k} \cdot \alpha_{\ell}\right) \cdot \alpha_{1} \ldots \widehat{\alpha_{k} \alpha_{\ell}} \ldots \alpha_{n}\right) \\
& +\frac{1}{2} \sum_{I \sqcup J=\{1, \ldots, n\}} \varepsilon_{\alpha}(I, J) Q_{2}^{\prime}\left(\mathcal{F}_{(|I|), m_{I}}\left(\alpha_{I}\right) \cdot \mathcal{F}_{(|J|), m_{J}}\left(\alpha_{J}\right)\right) \\
& =-\frac{1}{2}(\star)+\frac{1}{2}(\star \star) .
\end{aligned}
$$

On suppose que l'équation de fomalité est vérifiée jusqu'à l'ordre $n-1$ et on veut montrer que

$$
d_{H}\left(-\frac{1}{2}(\star)+\frac{1}{2}(\star \star)\right)=0 \quad \text { ou } \quad Q_{1}^{\prime}(\star)=Q_{1}^{\prime}(\star \star) .
$$

Comme $\delta \circ \delta=0$ et comme l'équation est vérifiée à l'ordre $n-1$,

$$
\begin{aligned}
Q_{1}^{\prime}(\star) & =\sum_{k \neq \ell} \varepsilon_{\alpha}(k \ell, 1 \ldots \widehat{k \ell} \ldots n) Q_{1}^{\prime}\left(\mathcal{F}_{(n-1), m}\left(Q_{2}\left(\alpha_{k} \cdot \alpha_{\ell}\right) \cdot \alpha_{1} \ldots \widehat{\alpha_{k} \alpha_{\ell}} \ldots \alpha_{n}\right)\right) \\
& =-\frac{1}{2} \sum_{k \neq \ell} \varepsilon_{\alpha}(k \ell, 1 \ldots \widehat{k \ell} \ldots n) \\
& \sum_{I \sqcup J=\{0,1, \ldots \widehat{k \ell}, \ldots, n\}} \varepsilon_{\tilde{\alpha}}(I, J) Q_{2}^{\prime}\left(\mathcal{F}_{(|I|), m_{I}}\left(\alpha_{I}\right) \cdot \mathcal{F}_{(|J|), m_{J}}\left(\alpha_{J}\right)\right) \\
=(1) &
\end{aligned}
$$

où on a posé $\alpha_{0}=Q_{2}\left(\alpha_{k} \cdot \alpha_{\ell}\right)$. D'autre part

$$
\begin{aligned}
Q_{1}^{\prime}(\star \star)= & -\sum_{I \sqcup J=\{1, \ldots, n\}} \varepsilon_{\alpha}(I, J) Q_{2}^{\prime}\left(Q_{1}^{\prime}\left(\mathcal{F}_{(|I|), m_{I}}\left(\alpha_{I}\right)\right) \cdot \mathcal{F}_{(|J|), m_{J}}\left(\alpha_{J}\right)\right) \\
& -\sum_{I \sqcup J=\{1, \ldots, n\}} \varepsilon_{\alpha}(I, J)(-1)^{m_{|I|}} Q_{2}^{\prime}\left(\mathcal{F}_{(|I|), m_{I}}\left(\alpha_{I}\right) \cdot Q_{1}^{\prime}\left(\mathcal{F}_{(|J|), m_{J}}\left(\alpha_{J}\right)\right)\right)
\end{aligned}
$$

car si $A_{1}$ est un opérateur $m_{1}$-différentiel et $A_{2}$ un opérateur multi-différentiel

$$
Q_{1}^{\prime} Q_{2}^{\prime}\left(A_{1} \cdot A_{2}\right)=-\left(Q_{2}^{\prime}\left(Q_{1}^{\prime}\left(A_{1}\right) \cdot A_{2}\right)+(-1)^{m_{1}} Q_{2}^{\prime}\left(A_{1} \cdot Q^{\prime}\left(A_{2}\right)\right)\right) .
$$

On peut maintenant utiliser l'hypothèse de récurrence pour calculer ces termes. Donc, en notant $[1, n]$ l'ensemble $\{1, \ldots, n\}, I_{\widehat{k \ell}}$ l'ensemble $I \backslash\{k, \ell\}$ et, si $K$ est 
une partie de $\{1, \ldots, n\}$, on notera abusivement $\varepsilon_{\alpha}(I, J)$ le nombre $\varepsilon_{\alpha_{K}}(I, J)$ si $I \sqcup J=K$, on aura :

$$
\begin{aligned}
& Q_{1}^{\prime}(\star \star)=-\frac{1}{2} \sum_{I \sqcup J=[1, n]} \varepsilon_{\alpha}(I, J) \sum_{k_{1} \neq \ell_{1} \in I} \varepsilon_{\alpha}\left(k_{1} \ell_{1}, I_{\widehat{k_{1} \ell_{1}}}\right) \\
& Q_{2}^{\prime}\left(\mathcal{F}_{(|I|), m_{|I|}+1}\left(Q_{2}\left(\alpha_{k_{1}} \cdot \alpha_{\ell_{1}}\right) \cdot \alpha_{I_{k_{1 \ell_{1}}}}\right) \cdot \mathcal{F}_{|J|, m_{J}}\left(\alpha_{J}\right)\right) \\
& +\frac{1}{2} \sum_{I \sqcup J=\{1, \ldots, n\}} \varepsilon_{\alpha}(I, J) \sum_{I_{1} \sqcup I_{2}=I} \varepsilon_{\alpha}\left(I_{1}, I_{2}\right) \\
& Q_{2}^{\prime}\left(Q_{2}^{\prime}\left(\mathcal{F}_{\left(\left|I_{1}\right|\right), m_{I_{1}}}\left(\alpha_{I_{1}}\right) \cdot \mathcal{F}_{\left(\left|I_{2}\right|\right), m_{I_{2}}}\left(\alpha_{I_{2}}\right)\right) \cdot \mathcal{F}_{(|J|), m_{J}}\left(\alpha_{J}\right)\right) \\
& -\frac{1}{2} \sum_{I \sqcup J=[1, n]} \varepsilon_{\alpha}(I, J)(-1)^{\left|k_{I}\right|} \sum_{k_{2} \neq \ell_{2} \in J} \varepsilon_{\alpha}\left(k_{2} \ell_{2}, J_{\widehat{k_{2} \ell_{2}}}\right) \\
& Q_{2}^{\prime}\left(\mathcal{F}_{(|I|), m_{I}}\left(\alpha_{I}\right) \cdot \mathcal{F}_{(|J|), m_{|J|}+1}\left(Q_{2}\left(\alpha_{k_{2}} \cdot \alpha_{\ell_{2}}\right) \cdot \alpha_{J_{k_{2} \ell_{2}}}\right)\right) \\
& +\frac{1}{2} \sum_{I \sqcup J=[1, n]} \varepsilon_{\alpha}(I, J)(-1)^{\left|k_{I}\right|} \sum_{J_{1} \sqcup J_{2}=J} \varepsilon_{\alpha}\left(J_{1}, J_{2}\right) \\
& Q_{2}^{\prime}\left(\mathcal{F}_{(|I|), m_{I}}\left(\alpha_{I}\right) \cdot Q_{2}^{\prime}\left(\mathcal{F}_{\left(\left|J_{1}\right|\right), m_{J_{1}}}\left(\alpha_{J_{1}}\right) \cdot \mathcal{F}_{\left(\left|J_{2}\right|\right), m_{J_{2}}}\left(\alpha_{J_{2}}\right)\right)\right) \\
& =-\frac{1}{2} \sum_{I \sqcup J=[1, n]} \varepsilon_{\alpha}(I, J) \sum_{k_{1} \neq \ell_{1} \in I} \varepsilon_{\alpha}\left(k_{1} \ell_{1}, I_{\widehat{k_{1} \ell_{1}}}\right) \\
& Q_{2}^{\prime}\left(\mathcal{F}_{(|I|), m_{|I|}+1}\left(Q_{2}\left(\alpha_{k_{1}} \cdot \alpha_{\ell_{1}}\right) \cdot \alpha_{I_{k_{1} \ell_{1}}}\right) \cdot \mathcal{F}_{(|J|), m_{J}}\left(\alpha_{J}\right)\right) \\
& -\frac{1}{2} \sum_{I \sqcup J=[1, n]} \varepsilon_{\alpha}(I, J)(-1)^{\left|k_{I}\right|} \sum_{k_{2} \neq \ell_{2} \in J} \varepsilon_{\alpha}\left(k_{2} \ell_{2}, J_{\widehat{k_{2} \ell_{2}}}\right) \\
& Q_{2}^{\prime}\left(\mathcal{F}_{(|I|), m_{I}}\left(\alpha_{I}\right) \cdot \mathcal{F}_{(|J|), m_{|J|}+1}\left(Q_{2}\left(\alpha_{k_{2}} \cdot \alpha_{\ell_{2}}\right) \cdot \alpha_{J_{k_{2 \ell_{2}}}}\right)\right) \\
& +\frac{1}{2} \sum_{I_{1} \sqcup I_{2} \sqcup I_{3}=[1, n]} \varepsilon_{\alpha}\left(I_{1} \sqcup I_{2}, I_{3}\right) \varepsilon_{\alpha}\left(I_{1}, I_{2}\right) \\
& Q_{2}^{\prime}\left(Q_{2}^{\prime}\left(\mathcal{F}_{\left(\left|I_{1}\right|\right), m_{I_{1}}}\left(\alpha_{I_{1}}\right) \cdot \mathcal{F}_{\left(\left|I_{2}\right|\right), m_{I_{2}}}\left(\alpha_{I_{2}}\right)\right) \cdot \mathcal{F}_{\left|I_{3}\right|, m_{I_{3}}}\left(\alpha_{I_{3}}\right)\right) \\
& +\frac{1}{2} \sum_{I_{1} \sqcup I_{2} \sqcup I_{3}=[1, n]} \varepsilon_{\alpha}\left(I_{1}, I_{2} \sqcup I_{3}\right)(-1)^{\left|k_{I_{1}}\right|} \varepsilon_{\alpha}\left(I_{2}, I_{3}\right) \\
& Q_{2}^{\prime}\left(\mathcal{F}_{\left(\left|I_{1}\right|\right), m_{I_{1}}}\left(\alpha_{I_{1}}\right) \cdot Q_{2}^{\prime}\left(\mathcal{F}_{\left(\left|I_{2}\right|\right), m_{I_{2}}}\left(\alpha_{I_{2}}\right) \cdot \mathcal{F}_{\left(\left|I_{3}\right|\right), m_{I_{3}}}\left(\alpha_{I_{3}}\right)\right)\right) \\
& =\left(1^{\prime}\right)+\left(2^{\prime}\right)+\left(3^{\prime}\right)+\left(4^{\prime}\right) \text {. }
\end{aligned}
$$


Il n'est pas difficile de voir que $\left(1^{\prime}\right)+\left(2^{\prime}\right)=(1)$ et $\left(3^{\prime}\right)=\left(4^{\prime}\right)=0$ d'après l'identité de Jacobi graduée. En effet pour cela il suffit de remarquer que

$$
\begin{aligned}
\varepsilon_{\alpha}(I, J) \varepsilon_{\alpha}(k \ell, I \backslash\{k, \ell\}) & =\varepsilon_{\alpha}(k \ell, I \backslash\{k, \ell\}, J) \\
& =\varepsilon_{\alpha}(k \ell, 1 \ldots \widehat{k \ell} \ldots n) \varepsilon_{\alpha}(I \backslash\{k, \ell\}, J) \\
& =\varepsilon_{\alpha}(k \ell, 1 \ldots \widehat{k \ell} \ldots n) \varepsilon_{\tilde{\alpha}}(\{0\} \sqcup I \backslash\{k, \ell\}, J)
\end{aligned}
$$

et

$$
\varepsilon_{\alpha}\left(I_{1} \sqcup I_{2}, I_{3}\right)=\varepsilon_{\alpha}\left(I_{1}, I_{2}, I_{3}\right) .
$$

Remarque. - Nos résultats prouvent que l'obstruction à l'existence d'une formalité sur $\mathbb{R}^{\bullet}$ se trouve localisée dans l'espace des vrais graphes d'ordre $1, \ldots, 1$, totalement antisymétriques. La cohomologie associée à l'opérateur $\delta$ restreint à cet espace de graphe doit permettre de contourner cette obstruction.

\section{BIBLIOGRAPHIE}

[1] Arnal (D.), Ben Amar (N.) \& Masmoudi (M.) - Cohomologiy of good graphs and Kontsevich linear star product, Lett. Math. Phys., t. 48 (1999), pp. 291-306.

[2] Arnal (D.), Manchon (D.) \& Masmoudi (M.) - Choix des signes pour la formalité de Kontsevich, Preprint arXiv:math. QA/0003003, mars 2000.

[3] Berger (R.) - Cohomologie différentiable des algèbres de polynômes, de leurs localisées ou de leurs complétées et des variétés, Publication du Département de Mathématiques de l'Université Claude Bernard-Lyon 1, nouv. série, vol. 5/A, p. 1-19, 1982.

[4] Kontsevich (M.) - Deformation quantization on Poisson manifolds I, Preprint q-Alg/97 09040, 1997. 